Z Herz- Thorax- Gefäßchir 2021 · 35:378-379 https://doi.org/10.1007/s00398-021-00467-6 Angenommen: 13. Oktober 2021 Online publiziert: 7. November 2021 ○ Der/die Autor(en) 2021

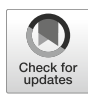

\section{Stella van Praagh (1927-2006), Richard van Praagh (geb. 1930)}

\author{
H. E. Ulmer \\ Heidelberg, Deutschland
}

Dieser Beitrag ist eine aktualisierte Fassung des Kapitels: Ulmer HE (2019) Meister ihres Fachs: Stella van Praagh, Richard van Praagh. In: Weil J, Kallfelz HC, Lindinger A, Schmaltz AA (Hrsg) Kinderkardiologie in Deutschland: 50 Jahre Deutsche Gesellschaft für Pädiatrische Kardiologie 1969-2019. Elsevier 2019, S. 340-341 (mit freundlicher Genehmigung des Elsevier Verlages).

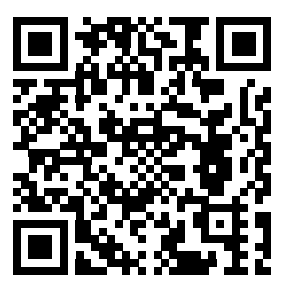

QR-Code scannen \& Beitrag online lesen
Die Beschäftigung mit dem Namen van Praagh im Zusammenhang mit der pädiatrischen Kardiologie führt früher oder später zwangsläufig zu zwei Menschen, deren wissenschaftliches Leben nahezu symbiotisch verlaufen ist und deren menschliche Verbundenheit von ihren Freunden gelegentlich als eine lebenslange Romanze bezeichnet wurde (- Abb. 1).

Richard van Praagh wurde als Sohn einer ursprünglich in Mähren und in den Niederlanden beheimateten Familie im April 1930 in London, Ontario in Kanada, geboren. Das Studium der Medizin in Toronto mussten sich Richard und sein Bruder lan, ein späterer Gynäkologe, durch die verschiedensten Jobs, u. a. auch als Totengräber, mitfinanzieren. In 1954 beendete Richard sein Studium an der jedoch erfolgreich mit dem Abschluss eines „MD“. Das „Postgraduate"-Training in Pädiatrie, Kardiologie und Pathologie dehnte sich dann über insgesamt 10 Jahre aus und führte von Toronto über Boston und die MayoClinic nach Baltimore. Hier wurde Richard eines Tages, im Anschluss an einen seiner Fortbildungsvorträge von einer jungen Assistentin nicht nur mit intelligenten Fragen überhäuft, sondern auch von „.... großen braunen Augen" fasziniert. - Dies sollte der Beginn einer letzten Endes 44 Jahre andauernden Romanze sein. 1962 heirateten Richard van Praagh und Stella Zacharioudaki.

Stella Zacharioudaki war die 1927 auf Kreta geborene Tochter einer Winzerfamilie. Sie absolvierte ihr Studium der Medizin in Athen und wurde dort als Jahrgangsbeste 1952 graduiert. Noch im selben Jahr emigrierte sie alleine, d. h. ohne ihre Familie, in die Vereinigten Staaten. Hier setzte sie ihre Ausbildung von Anfang an mit Interesse für angeborene Herzfehler in New Jersey, Buffalo und Baltimore fort, wo sie u. a. als Fellow bei Helen Taussig tätig war. 1962, zur Zeit ihrer Eheschließung mit Richard, war sie kardiologisches „staff member" in Buffalo, NY, während er mit dem Schwerpunkt Pathologie in den ersten drei Jahren ihrer Ehezunächst in Toronto, später in Chicago tätig war. Bemerkenswerterweise wurden innerhalb dieser drei Jahre alle drei Kinder der jungen Familie geboren.

In dieser Zeit der rapiden Entwicklung der neuen Subspezialität, der pädiatrischen Kardiologie, in den frühen 1960erJahren, war eines der aufkommenden Probleme in diesem Bereich das Fehlen einer einheitlichen Nomenklatur und einer Klassifikation für angeborene Herzfehler, wodurch die Kommunikation und der wissenschaftliche Austausch hierüber unsinnigerweise erheblich erschwert waren. Während Richard bereits früh damit be-

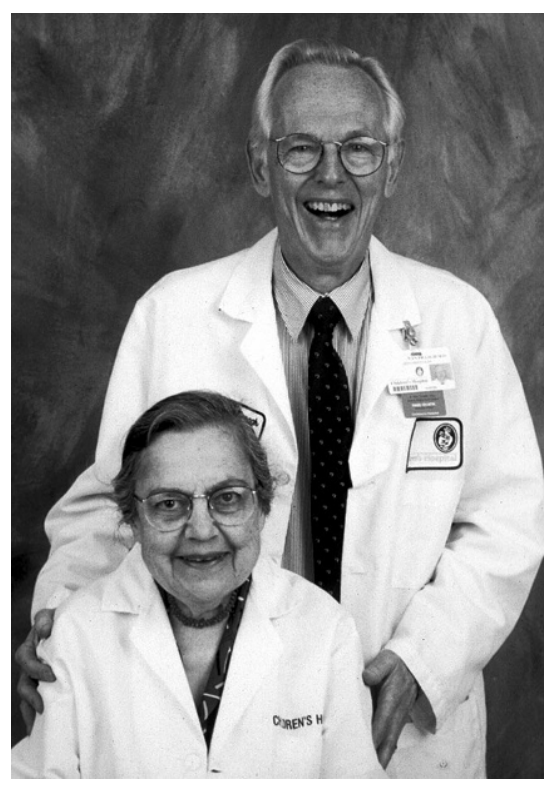

Abb. $1 \Delta$ Richard und Stella van Praagh. (Aus Van Praagh [1]. @ 2012 Society for Pediatric Pathology. Mit freundl. Genehmigung von SAGE Journals.) 
gonnen hatte, ein derartiges System zum eigenen Gebrauch zu entwickeln, gelang es Stella durch dessen Anwendung bei ihrer eigenen Arbeit, damit darzustellen, dass es sich bei der Fallot-Tetralogie nicht, wie bisher angenommen, um das zufällige gemeinsame Vorkommen von vier einzelnen Defekten, sondern um eine einzige, entwicklungsgeschichtlich bedingt zusammengehörige Malformation des Herzen handeln musste.

Es nimmt daher nicht wunder, dass Alexander Nadas die Gelegenheit wahrnahm, beide Wissenschaftler, Stella und Richard van Praagh, an das Children's Hospital der Harvard University nach Boston zu berufen, zur Entwicklung des von inm neu installierten „Pediatric-cardiology“Programms. Er übertrug ihnen die Leitung der "cardiac registry“. Die sich daraus entwickelnde, außerordentlich fruchtbare Tätigkeit übten beide gemeinsam und ununterbrochen über nahezu 40 Jahre mit großem Erfolg aus, von 1965 bis zu ihrer ebenfalls gemeinsamen Emeritierung 2002.

Richard van Praagh hatte bereits 1964 erstmals sein System eines "segmentalen Situs" zur Beschreibung der Morphologie komplexer angeborener Herzfehler, z.B. bei der Dextrokardie, vorgestellt und es 1972 in seiner grundlegenden Arbeit "The Segmental Approach to Diagnosis of Congenital Heart Disease" publiziert, jetzt jedoch erweitert zur Anwendung auf alle angeborenen Herzfehler. Das zentrale Prinzip beruht auf der Unterteilung des Herzens in drei Segmente, die Vorhöfe, die Kammern und die großen Gefäße, sowie ihrer räumlichen Beziehung zueinander.

Etwa zur gleichen Zeit entwickelte Robert Anderson in Großbritannien ebenfalls ein segmentales Ordnungsprinzip des Herzens, wobei für inn jedoch nicht die Position des "segmentalen Situs" auschlaggebend war, sondern die "sequenzielle Konnexion" der einzelnen Segmente des Herzens. Diese beiden Schulen der kardialen Nomenklatur waren trotz einiger Ähnlichkeiten anfangs stark polarisiert und wurden in ihren Differenzen von ihren Vätern heftig umkämpft, ohne dass jedoch die gegenseitige Achtung der beiden Kontrahenten darüber in die Brüche gegangen wäre. Es bedurfte allerdings der Gründung einer eigenen „International Society for
Nomenclature of Paediatric and Congenital Heart Disease" 2005 mit der Mission einer Vereinheitlichung der verschiedenen Nomenklaturen in einen einzigen Code. Eine erste Version hiervon erschien dann erstmals 2015.

Stella und Richard van Praagh veröffentlichten in 40 Jahren gemeinsam über 200 umfangreiche wissenschaftliche Originalarbeiten sowie Bücher und Beiträge in Lehrbüchern, was Stella in der Ansprache zu ihrer gemeinsamen Emeritierung 2002 zu der augenzwinkernden Bemerkung veranlasste: "Dickie, you and I have proved that a husband and a wife really can work together." Richard und Stella führten auch danach ein offenes und außerordentlich gastfreundliches Haus, das sowohl für Stellas heimatliche Backkünste und griechischen Wein bekannt war als auch wegen einer hohen menschlichen und philosophischen Gesprächskultur geschätzt wurde.

Gemeinsam erhielten beide 1999 den Award for Cardiovascular Pathology und wurden 2004, ebenfalls gemeinsam, mit dem Paul Dudley White Award der American Heart Association ausgezeichnet.

Am 3. Juni 2006 verstarb Stella van Praagh, drei Tage nach einem akuten Schlaganfall. Richard van Praagh war es zumindest bis 2015 noch möglich, trotz schwerer eigener Erkrankung weiterhin Einladungen auf internationale Kongresse anzunehmen und in seinem unvergleichlichen Ostküstenstil anregende Übersichtsvorträge zu halten.

\section{Korrespondenzadresse}

\section{Prof. Dr. H. E. Ulmer}

Am Aukopf 5, 69118 Heidelberg, Deutschland herbert_ulmer@t-online.de

\section{Literatur}

1. Van Praagh R (2012) The Farber-Landing Lecture: Pediatric Pathology - the Clinician's “Open Sesame" and its Importance in Pediatric Cardiology and Cardiovascular Surgery. Pediatr Dev Pathol 15(6):431-449. https://doi.org/10.2350/10-100924-OA.1

\section{Autoren WERKSTATT \\ GRATIS \\ Ein Service für Autoren von Springer Medizin S \\ Fortbildungen für Autor*innen und Gutach- ter*innen}

Die ersten Veröffentlichungen sind für Jeden ein wichtiger Schritt in der angestrebten Karriere. Wissenschaftliche Artikel sind entscheidend dafür, dass die eigene Arbeit in der Community wahrgenommen wird. Es geht darum, die eigenen Ideen national und international auszutauschen und sicherzustellen, dass die Ergebnisse Wirkung erzielen.

Die Online-Kurse der Autorenwerkstatt helfen, sich leicht einen Überblick über das Schreiben, Einreichen, Begutachten und Veröffentlichen eines Manuskripts zu verschaffen.

5 Online-Kurse zu den wichtigsten Standards des wissenschaftlichen Publizierens:

- Wie verfasse ich ein Manuskript?

- Writing in English für deutschsprachige Autorinnen und Autoren

- Wie funktionieren Publikation und Begutachtung?

- Anleitung zur Open-AccessVeröffentlichung

- Leitfaden zur Peer-ReviewBegutachtung

Für alle, die auf SpringerMedizin.de registriert sind!

Jetzt gratis fortbilden unter www.springermedizin.de/ autorenwerkstatt/ 